\title{
Silicon fibre nano-spike for robust coupling to silica fibres
}

\author{
H. Ren ${ }^{1}$, A. F. J. Runge ${ }^{1}$, J. Campling ${ }^{1}$, M. Jones ${ }^{2}$, T. Hawkins ${ }^{2}$, J. Ballato ${ }^{2}$, P. Horak ${ }^{1}$, U. Gibson ${ }^{3}$, \\ and A. C. Peacock ${ }^{1}$ \\ 1. Optoelectronic Research Centre, University of Southampton, SO17 1BJ, UK \\ 2. COMSET, School of Materials Science and Engineering, Clemson University, Clemson, South Carolina 29634, USA \\ 3. Department of Physics, Norwegian University of Science and Technology, 7491 Trondheim, Norway
}

Making use of the attractive properties of silicon (Si), a wide array of highly functional photonic devices have been demonstrated over the past few decades [1]. Although planar platforms are still the most prominent choice for integrated systems, more recently silicon fibres have gained increased attention due to their simple fabrication methods and flexible device designs [2]. However, despite their physical similarity to traditional single-mode fibres (SMFs), coupling light efficiently into and out of these devices remains a difficult task due to the high refractive index of the Si core, which results in significant reflection losses and a large mode mismatch. A few solutions have been proposed to overcome this integration challenge, including the use of microstructured fibre designs to better match the mode area [2] and chemical etchants to reduce the reflection at the interface [3], though so far no method has addressed both issues simultaneously. Thus a promising alternative solution would be to adapt the well-established inverse taper approach widely employed by the planar community [4], which is also gaining attention within the novel material fibre community [5]. The key idea behind this approach is that by decreasing the Si core to nanoscale dimensions at the facet, the guided mode spreads out to better match both the area and the effective index of the SMF mode. In this paper, we demonstrate the fabrication and optical characterization of the first Si fibre nano-spike which has been directly spliced to SMF.

The starting Si fibre was fabricated using a modified fibre drawing technique [6]. To fabricate the nano-spike, the fibre is subsequently tapered using the method described in [7]. However, in this instance the filament power is adjusted to cause a break-up of the Si core, which can be controlled to form a continuous transition from the untapered region down to the tip of the spike, as shown in Fig. 1(a). The fibre is then cleaved and spliced to a SMF using a conventional splicing method. An example of a typical integrated SMF-Si fibre device is shown in Fig. 1(b). Finally, the co-joined fibres are inserted into a thick capillary, fixed with wax to provide mechanical robustness, so that the Si fibre facet can be polished.

The optical transmission properties of the integrated fibres were then characterized using a $1550 \mathrm{~nm}$ c.w. laser. A connector was spliced to the SMF side of the device while a microscope objective was used to collect the light from the Si core. It is clear from the output mode, shown in the inset of Fig. 1(b), that the light coupled from the SMF is well confined in the core. Taking into account the loss contributions from the free space coupling and the Si material, we place an upper bound on the coupling loss to be $\sim 9 \mathrm{~dB}$ from SMF to Si. Although this loss value is currently higher than our free space method ( 7-8 dB for a few micron core Si fibre), we attribute this to the non-optimized dimensions of the nano-spike and a misalignment of the fibre cores due to a mismatch in their outer diameters, leaving considerable room for improvement. Specifically, calculations of the mode overlap between the SMF and the Si nano-spike as a function of the tip diameter, as shown in Fig. 1(c), reveal that the maximum transmission between the two fibres could reach as high as $\sim 0.5 \mathrm{~dB}$ for an optimized tip diameter of $190 \mathrm{~nm}$. Thus we believe that this nano-spike splicing approach provides a clear route to the development of robust and practical integrated Si fibre devices, of use for wide ranging optical and optoelectronic applications.
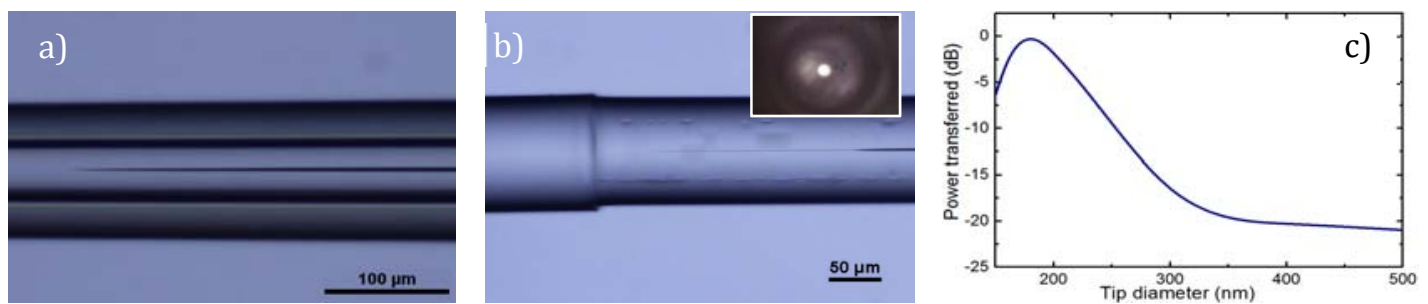

Fig. 1 Microscope image of the Si nano-spike (a) and the device spliced to SMF (b). The inset shows the output mode from the Si core. (c) Mode overlap between SMF and Si nano-spike as a function of tip diameter.

\section{References}

[1] B. Jalali and S. Fathpour, “Silicon Photonics,” J. Lightwave Technol. 24, 4600 (2006).

[2] A. C. Peacock, J. R. Sparks, and N. Healy, “Semiconductor optical fibres: progress and opportunities,” Laser Photon. Rev. 8, 53 (2014).

[3] J. Chen, Y. Sun, and L. A. Wang, “Reducing splicing loss between a silicon-cored optical fiber and a silica optical fiber,” IEEE Photon. Tech. Lett. 28, 1774 (2016).

[4] V. R. Almeida, R. R. Panepucci, and M. Lipson, “Nanotaper for compact mode conversion,” Opt. Lett. 28, 1302 (2003).

[5] N. Granzow, M. A. Schmidt, W. Chang, L. Wang, Q. Coulombier, J. Troles, P. Toupin, I. Hartl, K. F. Lee, M. E. Fermann, L.

Wondraczek, and P. St. J. Russell, “Mid-infrared supercontinuum generation in $\mathrm{As}_{2} \mathrm{~S}_{3}$-silica "nanospike” step-index waveguide,” Opt.

Express 21, 10969 (2013).

[6] E. F. Nordstrand, A. N. Dibbs, A. J. Eraker, and U. J. Gibson, “Alkaline oxide interface modifiers for silicon fiber production,” Opt. Mat. Express 3, 651 (2013).

[7] F. Shuailin. L. Shen, N. Healy, L. Xiao, M. Jones, T. Hawkins, J. Ballato, U. Gibson and, A. C. Peacock, “Tapered polysilicon core fibers for nonlinear photonics” Opt. Lett. 41, 1360 (2016). 